\title{
Retinoids Increase Lung Elastin Expression But Fail to Alter Morphology or Angiogenesis Genes in Premature Ventilated Baboons
}

\author{
RICHARD A. PIERCE, BELINDA JOYCE, SUSAN OFFICER, CATHERINE HEINTZ, CARLA MOORE, DONALD MCCURNIN, \\ CARL JOHNSTON, AND WILLIAM MANISCALCO
}

\begin{abstract}
Division of Pulmonary and Critical Care Medicine [R.A.P., B.J., S.O., C.H., C.M.], Washington University School of Medicine, St. Louis, Missouri 63130; Department of Pediatrics [D.M.], University of Texas Health Science Center; San Antonio, Texas 78229; Strong Children's Research Center [C.J., W.M.], University of Rochester School of Medicine and Dentistry, Rochester, New York 14642
\end{abstract}

\begin{abstract}
Retinoids regulate elastin synthesis by alveolar myofibroblasts and affect angiogenesis pathways, both of which are processes critical for alveolar development. Retinoids accelerate alveolarization in rodents and are now used therapeutically in premature infants at risk of bronchopulmonary dysplasia (BPD). This study examined the effects of retinoid supplementation on alveolar elastin expression and deposition and angiogenesis-related signaling in a primate model of BPD. Premature baboons delivered at $125 \mathrm{~d}$ of gestation after maternal steroid treatment were given surfactant and ventilated with minimal supplemental oxygen for $14 \mathrm{~d}$ with $(n=5)$ and without $(n=5)$ supplemental vitamin A $(5000 \mathrm{U} / \mathrm{kg} / \mathrm{d})$ and compared with 140-d unventilated controls. Ventilatory efficiency index (VEI) and oxygenation index (OI) were not statistically different between ventilated treatment groups. Expression of vascular endothelial growth factor A (VEGF-A), fms-related tyrosine kinase 1 (Flt-1), and tyrosine kinase with immunoglobulin-like and EGF-like domains 1 (TIE-1) was repressed by premature delivery and mechanical ventilation and was not altered by retinoid supplementation. Retinoid supplementation did not enhance alveolar angiogenesis. Elastin expression was repressed by premature delivery and extended ventilation, and retinoid supplementation increased elastin expression specifically in alveolar myofibroblasts within alveolar walls. These results suggest that the small decrease in mortality among premature infants receiving retinoid supplementation may not be mediated through enhanced alveolar development. (Pediatr Res 61: 703-709, 2007)
\end{abstract}

$\mathbf{M}^{2}$ any premature infants develop respiratory distress and often require mechanical ventilation and supplemental oxygen to survive. These interventions can perpetuate injury and ultimately lead to altered lung architecture BPD (1), with failed alveolar development, which is a hallmark of BPD (2). The incidence of BPD remains high in infants of very low birth weight despite advancements such as surfactant therapy.

The findings that retinoid signaling is required for alveolar development in mice and that retinoid treatment can enhance alveolar development in rats (3-5) led to vitamin A supple-

Received November 13, 2006; accepted February 8, 2007.

Correspondence: Richard A. Pierce, Ph.D., Washington University School of Medicine, Campus Box 8052, 660 South Euclid Ave., St. Louis, MO 63130; e-mail: rpierce@im. wustl.edu

Supported by NIH-5U01HL063387-06 (R.P.) and 5U01HL063400-07 (W.M.).

DOI: $10.1203 /$ pdr.0b013e318053661d mentation of preterm infants at risk of BPD. Some clinical trials of vitamin A supplementation in premature infants have shown no benefit, and others have found that dose is critical (6-8). Tyson et al. (9) found that overall, one additional infant survived without chronic lung disease for every $14-15$ infants who received vitamin A supplements . The mechanism by which vitamin A supplementation may reduce BPD or mortality is not well defined, but may be linked to enhanced alveolar development.

The altered alveolar capillary network associated with BPD (10-12) supports the "vascular hypothesis" that failed capillary development is central to the pathogenesis of BPD (13). Retinoid signaling is required for alveolar capillary development. Vitamin A supplementation may decrease the incidence of BPD by stimulating VEGF expression in alveolar epithelial cells $(11,14)$ or through other proangiogenic pathways $(15)$. Retinoid signaling also increases lung endothelial proliferation (16).

Vitamin A supplementation may also stimulate alveolar development by enhancing elastin expression or deposition by alveolar myofibroblasts, which are retinoid responsive and dependent $(17,18)$. The mechanical properties of distensibility and recoil of alveoli, facilitating influx of fresh air and exhalation of carbon dioxide from respiration, are conferred by elastic fibers encircling the openings of alveoli. Mice with genetic ablation of genes required for elastic fiber assembly or elastin expression have failed alveolar development (19).

To test the effects of retinoid supplementation on the incidence of BPD, alveolar capillary development, and alveolar elastic fiber deposition, we used an experimental model of BPD in the baboon (term $=185 \mathrm{~d}$ ). This model uses antenatal steroid therapy before premature delivery, surfactant therapy, and mechanical ventilation of premature baboons delivered at $125 \mathrm{~d}$ of gestation, with supplemental oxygen only as required. Retinoid supplementation stimulated alveolar elastin

\footnotetext{
Abbreviations: $\alpha$-SMA, $\alpha$-smooth muscle actin; BPD, bronchopulmonary dysplasia; FLT-1, fms-related tyrosine kinase 1; GAPDH, glyceraldehyde3-phosphate dehydrogenase; PECAM-1, platelet/endothelial cell adhesion molecule 1; RPA, ribonuclease protection assay; TIE-1, tyrosine kinase with immunoglobulin-like and EGF-like domains 1; VEI, ventilatory efficiency index
} 
expression but not alveolar angiogenesis, capillary development, or alveolar size in this study.

\section{MATERIALS AND METHODS}

Animals. Animal treatments were performed at the Southwest Foundation for Biomedical Research (San Antonio, TX) in accordance with the National Research Council's Guide for the Care and Use of Laboratory Animals. Fetal baboons are delivered by hysterotomy at $125 \pm 2 \mathrm{~d}$ or $140 \pm 2 \mathrm{~d}$ of gestation after antenatal steroid therapy (betamethasone $6 \mathrm{mg}$ at $48 \mathrm{~h}$ and $24 \mathrm{~h}$ before delivery). This is approximately equivalent to $27 \mathrm{wk}$ of gestation in humans. After delivery, baboons were intubated, treated with surfactant (Survanta at 4 $\mathrm{mg} / \mathrm{kg}$ ), and placed on positive pressure ventilation with tidal volumes of 4-6 $\mathrm{mL} / \mathrm{kg}$ to maintain $\mathrm{PCO}_{2}$ of $45-55 \mathrm{~mm} \mathrm{Hg}$ and supplemental $\mathrm{O}_{2}$ as needed to maintain arterial $\mathrm{Po}_{2}$ at $40-60 \mathrm{~mm} \mathrm{Hg}$ for $14 \mathrm{~d}$. Proper fluid supplementation, nutrition, and antibiotic support were provided. Ventilatory management, hemodynamic support, pulmonary function testing, and postmortem pressurevolume measurements were conducted as described (20). Five animals received daily retinol supplementation $(5 \mathrm{mg} / \mathrm{kg}$, Aquasol A delivered by intramuscular injection), and five received vehicle injections alone. Gestational control animals included premature baboons delivered at $140 \mathrm{~d}$ (5). At the time of killing by intravenous infusion of excess sodium pentobarbital sodium, the lungs were cleared by infusion of phosphate-buffered saline (PBS) in the right heart, and then lung tissue was snap-frozen in liquid nitrogen or inflated with $4 \%$ paraformaldehyde at $20 \mathrm{~cm}$ pressure for fixation.

Morphometry. Two sections from each of one to two blocks of right upper lung lobe tissue per animal in the study were stained with hematoxylin and eosin. Ten digital images at $200 \times$ magnification per section were captured using a random starting point and a random number generator, and then analyzed for chord length using NIH Image software (Version 1.63, National Institutes of Health, Bethesda, MD) with a chord length macro (available at http://rsb.info.nih.gov/nih-image) (21). Tissue density was calculated by assessing air space and tissue using Image Pro Plus software and the formula: Tissue density $=$ area occupied by tissue/total area (tissue + air space).

Ribonuclease protection assay (RPA). RPA were performed as described previously (11). The hAngio-1 riboprobe template (Pharmingen, San Diego, CA) was used to generate labeled antisense riboprobes. After electrophoresis, the intensity of each specific message band was measured using a computerlinked PhosphoImager with ImageQuant software (Molecular Dynamics) and normalized to the intensity of the constitutively expressed housekeeping gene L32.

Platelet/endothelial cell adhesion molecule 1 (PECAM-1) immunohistochemistry. PECAM-1 immunohistochemistry was performed with antigen retrieval in $50 \mathrm{mM}$ Tris- $\mathrm{HCl}, \mathrm{pH} 9.5$ with microwave treatment (11). The slides were treated with $\mathrm{H}_{2} \mathrm{O}_{2}$ to block endogenous peroxidase activity and blocked in triethanolamine-buffered saline (TBS) $+3 \%$ horse serum. Sections were incubated overnight with primary goat anti-PECAM-1 polyclonal antibody (Santa Cruz, sc-1506) and diluted 1:1200 in TBS/1.5\% horse serum at $4^{\circ} \mathrm{C}$. Biotinylated horse anti-goat IgG diluted $1: 200$ in TBS $/ 1.5 \%$ horse serum was added for $45 \mathrm{~min}$, followed by ABC-AP reagent. Chromogenic substrate was added, and the slides were incubated for 15-20 min. Slides were counterstained with hematoxylin and eosin. The negative control was goat $\mathrm{IgG}$ in $\mathrm{TBS} / 1 \%$ horse serum.

Hart's elastin staining. Rehydrated peripheral lung tissue sections $(5 \mu \mathrm{m})$ were soaked in $0.25 \%$ potassium permanganate solution for $5 \mathrm{~min}$, cleared in $5 \%$ oxalic acid, and soaked in resorcin-fuchsin solution (Poly Scientific, Bay Shore, NY) overnight. After washing in water, sections were counterstained with tartrazine (yellow), dehydrated through graded ethanol, cleared in xylene, and mounted.

Staining for $\alpha$-smooth muscle actin ( $\alpha$-SMA). Immunofluorescent staining for $\alpha$-SMA of rehydrated sections used overnight incubation at $4{ }^{\circ} \mathrm{C}$ with the primary antibody (1A4 mouse monoclonal, 1:200 dilution, Sigma Chemical Co., St. Louis, MO) after blocking with 10\% normal horse serum. After PBS washes, sections were incubated in the dark with secondary antibody (fluorescein-conjugated goat anti-mouse IgG, 1:250 dilution, $1 \mathrm{~h}$, room temperature; Jackson ImmunoResearch, West Grove, PA). After PBS washes, sections were viewed under fluorescent light for image capture.

Quantification of elastic fiber density and $\alpha-S M A$ staining. A minimum of 10 random lung fields of stained sections per animal at $200 \times$ magnification were captured with a digital camera and imported into Image Pro Plus software. For quantification of elastic fiber density, the proportion of the area of each image that was brown to black (elastic fibers) and yellow (nonelastic tissue) was determined with Image Pro Plus software (Media Cybernetics, San Diego, CA). For $\alpha$-SMA staining, the areas of saccular or alveolar lung tissue with each of three nonoverlapping intensity levels of staining for $\alpha$-SMA were determined. Images were captured with the same exposure to control for signal intensity. Statistical analysis was performed using the $t$ test for independent samples, two-tailed analysis.

Quantitative reverse transcriptase polymerase chain reaction (RT-PCR) for elastin expression. Total RNA isolated from peripheral lung was used to prepare cDNA using Superscript Plus RT and random hexamer priming. The cDNA was then subjected to PCR amplification for elastin and glyceraldehyde-3-phosphate dehydrogenase (GAPDH) as an internal control. Primers used were $5^{\prime}$ gtg ctg gtg ttc ctg gac tt $3^{\prime}$ as the ELN forward primer and $5^{\prime}$ agg cac tgc tgc tcc ata tt $3^{\prime}$ for the ELN reverse primer, yielding a 205-bp product, and $5^{\prime} \operatorname{tgc}$ acc acc aac tgc tta gc $3^{\prime}$ as the GAPDH forward primer and $5^{\prime}$ ggc atg gac tgt ggt cat gag3' for the GAPDH reverse primer, yielding a 104-bp product with a Stratagene MX 3000 instrument. Standard curves were generated, and results using Sybr Green for detection were standardized using reference cDNA for each run.

In situ hybridization. ${ }^{35} \mathrm{~S}$-labeled antisense or sense riboprobes were generated from a baboon tropoelastin cDNA linearized for in vitro transcription (Promega, Madison, WI). Rehydrated sections of peripheral lung were digested with proteinase $\mathrm{K}$, acetylated, and then hybridized with denatured radiolabeled riboprobe $(40,000 \mathrm{cpm} / \mu \mathrm{L})$ overnight at $60^{\circ} \mathrm{C}$. After stringent washes including digestion with RNase A, slides were exposed for $11 \mathrm{~d}$ and then developed and counterstained with hematoxylin and eosin.

\section{RESULTS}

Clinical outcomes: study groups and clinical course. There were no deaths in the control animals $(n=5)$ and one death in the retinoid-treated group $(n=5)$ due to respiratory failure and unresponsive hypotension. The gender distribution, gestational ages, and birth weights in the two treatment groups were similar. Daily fluid intake, daily urine output, and daily weights over the course of the study were similar between groups.

Pulmonary function. Protocols for ventilation and oxygen support were strictly guided by predetermined goals for blood gas values. The oxygenation index (OI) (Fig. 1A) and ventilatory efficiency index (VEI) (Fig. 1B) were similar for both the experimental groups. There was a trend toward reduced lung volumes achieved at the same inspiratory pressures postmortem in the retinoid-treated group (Fig. 1C). Lung compliance, as measured using a VitalTrends machine, was not statistically different between treatment groups (Fig. 1D).

Morphology. The 140-d gestational control lungs exhibit an alveolar appearance as previously described in this model (22) (Fig. 2A). Alveolar walls were relatively thin and had numerous septal projections (Fig. 2B). By comparison, lungs from animals delivered at $125 \mathrm{~d}$ and ventilated for $14 \mathrm{~d}$ displayed enlarged terminal air spaces with extended walls and a lack of septation in the untreated (Fig. 2C) and retinoid-treated (Fig. $2 E$ ) animals. In other regions of the same lungs, thickened hypercellular saccular walls were found in the untreated (Fig. $2 D$ ) and retinoid-treated (Fig. $2 F$ ) groups. The varied morphology in the ventilated groups was reflected in the greater variance in chord length compared with gestational controls. The mean chord length showed a trend toward increase with ventilation but did not achieve statistical significance. However, when the frequency of classes of chord lengths was assessed between groups, the retinoid-treated lungs showed an increase in the largest sized terminal air spaces compared with gestational controls.

Expression of angiogenesis-related genes. Because retinoic acid increases fetal lung endothelial cell proliferation (23) and may modulate VEGF expression (24), we analyzed mRNA expression of several angiogenesis-related genes by 
A
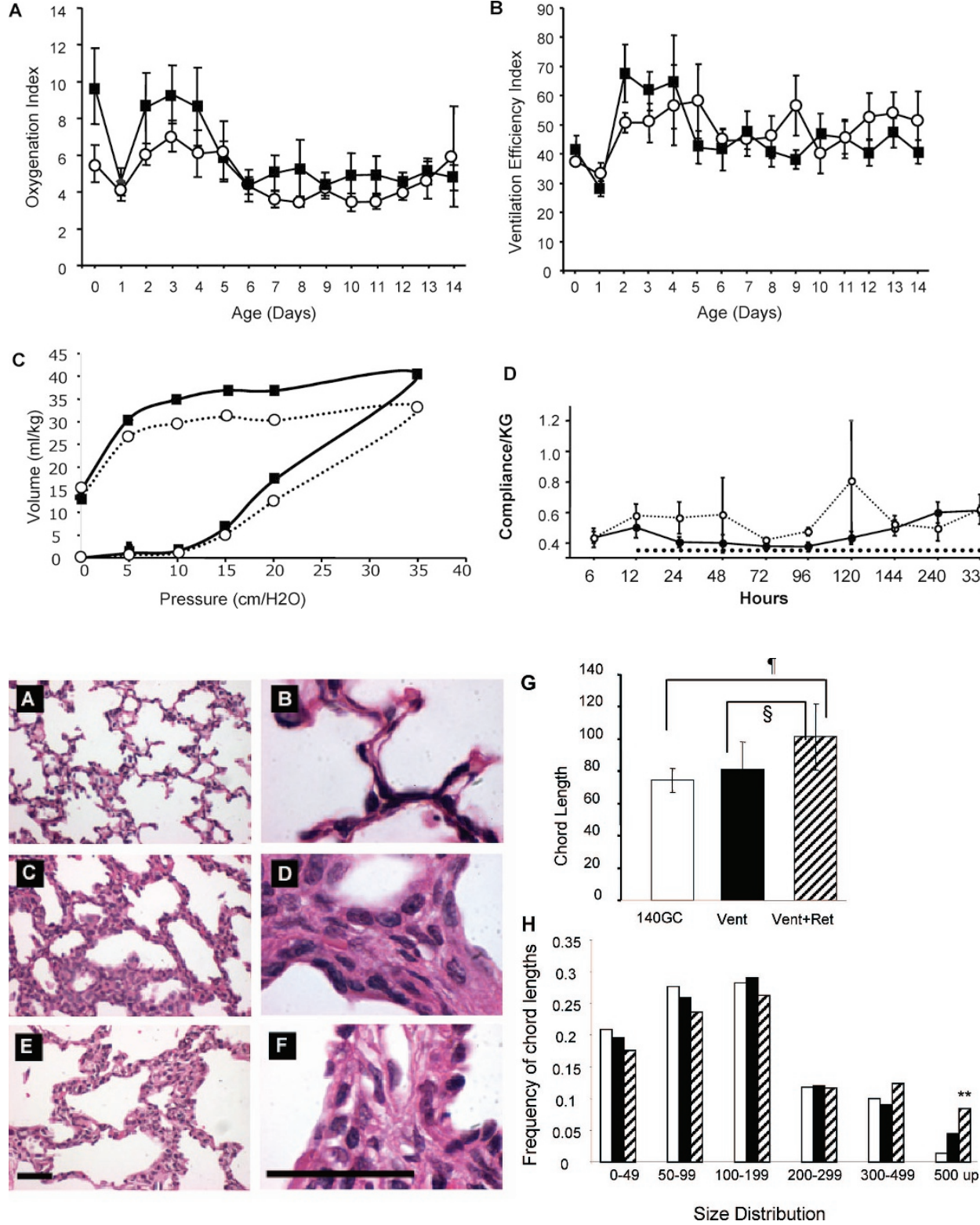

Figure 1. Physiologic effects of retinoid treatment. Shown are values for individual animals. Ventilation only (ם). Ventilation with retinoid supplementation $(\square)$. (A) $\mathrm{OI}=$ $\mathrm{FiO}_{2} * 100 * \mathrm{Paw} / \mathrm{PaO}_{2}$. (B) VEI $=\mathrm{PaCO}_{2}$ *rate*PIP/1000. (C) Postmortem pressurevolume analysis. $(D)$ Dynamic compliance measurements from throughout the course of treatment did not show statistical differences. Control $(\bigcirc)$; retinol $(\bullet)$.

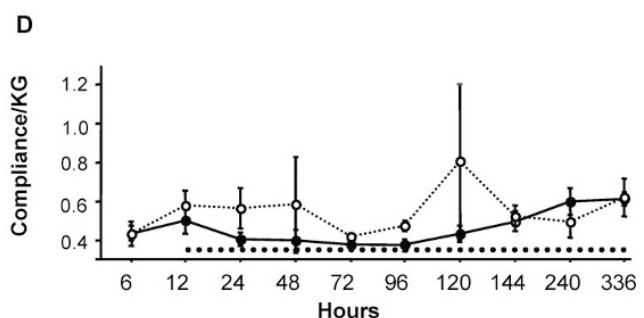

Figure 2. Morphology of premature baboon lung. Hematoxylin and eosin-stained panels at $200 \times(A, C, E)$ and $1000 \times(B, D, F) .(A$, $B)$ At $140 \mathrm{~d}$, gestational control lungs exhibit alveolar morphology. $(C, D)$ Premature baboons delivered at $125 \mathrm{~d}$ and ventilated for $14 \mathrm{~d}$ exhibit simplified, enlarged air spaces lacking alveolar wall extensions $(C)$ and thickened saccular walls $(D)$. Lungs from the retinoid supplementation group $(\mathbf{E}$, $F$ ) show similar developmental deformities. Bar $=100 \mu \mathrm{M} .(G, H)$ 140-d gestational control (open columns), ventilation-only (solid columns), and retinol-treated (hatched columns) animals.
RPA (Fig. 3). VEGF, FLT-1, and TIE-1 were significantly decreased in both treatment groups compared with the 140-d gestational controls.

PECAM-1 staining. To assess capillary development, sections of peripheral lung were stained for the endothelial marker PECAM-1 (Fig. 4A-C). As reported previously, the 140-d gestational control animals had a well-developed distal capillary network with many capillaries closely apposed to the terminal epithelium of thin inter-air space septa (11). The ventilated untreated animals often had capillaries embedded in the thickened saccular walls and occasional dilated capillaries, as noted previously in this BPD model (11). The retinoidtreated animals had capillary networks that were very similar to the untreated animals.

ELN fiber staining. We assessed elastic fiber staining in the alveolar compartment of the lung, as opposed to whole lung sampling of desmosine, which would include elastin within blood vessels and conducting airways as well as in the pleural surface. Dense elastic fibers localized to the tips of emerging alveolar septae in 140-d gestational lungs (Fig. 5A), and little elastic fiber staining was found within alveolar walls. In lungs of untreated ventilated animals, dense elastic fibers localized to shortened alveolar extensions and short brushlike elastic fibers were deposited along some saccular walls (Fig. 5B). In lungs of retinoid-treated ventilated animals, elastic fibers often stained more intensely and extended along walls of simplified terminal air spaces (Fig. 5C).

Lung elastin expression in lungs of retinoid-treated, ventilated premature baboons. Because retinoids stimulate expression of elastin in alveolar myofibroblasts, we examined elastin mRNA expression by quantitative reverse transcriptase polymerase chain reaction (RT-PCR) (Fig. 6). Elastin expression was increased 4.7-fold with retinoid treatment.

Localization of increased elastin expression. The localization of elastin mRNA expression was assessed by in situ hybridization. Elastin expression was intense in the muscular walls and endothelium of the largest intralobar pulmonary arteries in all treatment groups (Fig. 7A-F). Elastin expression 

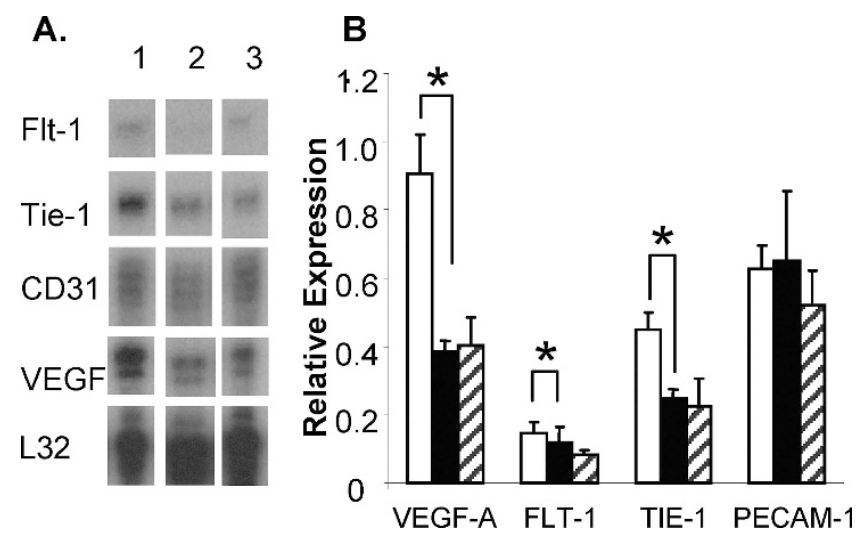

Figure 3. (A) Representative RPA gel for several genes in angiogenesis cluster. (B) Quantification of RPA data showing decreased Flt-1, TIE-1, and VEGF in ventilation-only animals compared with 140-d gestational control animals. There were no differences between the ventilation-only animals and the retinol-treated animals. 140-d gestational control (open columns), ventilation-only (solid columns), retinol-treated (hatched columns) animals. (Four to five animals for each condition. $* p<0.05$. Data are means \pm SEM.)

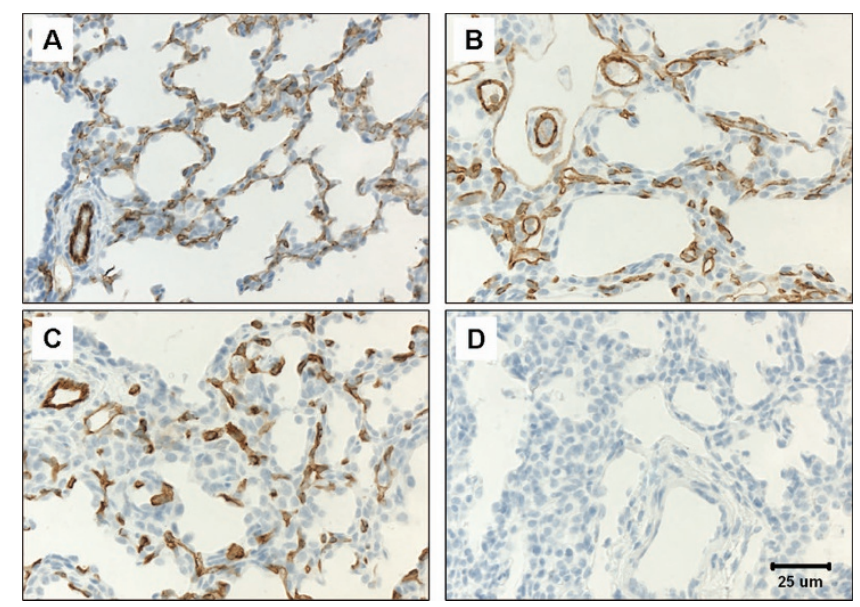

Figure 4. PECAM-1 immunohistochemistry. Representative fields from 140-d gestational control $(A)$, ventilation-only $(B)$, and retinol-treated $(C)$ animals and control antibody $(D)$. The 140-d gestational control animals had an extensive network of distal air space capillaries (brown staining) that were largely subepithelial. The capillary network in the ventilation-only animals appeared fragmented with isolated capillaries in thickened mesenchyme. The retinoid-treated animals were similar to the ventilation-only animals.

was detected in adventitial layers in retinoid-treated premature ventilated animals (Fig. $7 F$ ) but not in other treatment groups. Large airway smooth muscle exhibited abundant elastin expression in 140-d gestational controls (Fig. $7 G$ and $H$ ) was slightly increased in the ventilation-only group (Fig. $7 I$ and $J$ ) and the retinoid-treated group (Fig. $7 K$ and $L$ ). Signal for elastin mRNA in small airway smooth muscle was modest in all treatment groups (Fig. 7M-R), but vascular elastin expression was high in the same sections. A marked increase in elastin expression was found with retinoid treatment in the alveolar compartment of the lung (Fig. $7 S-X$ ). In alveolar walls of control animals, few cells expressed elastin at high levels (Fig. $7 S$ and $T$ ). In the ventilated group, intense expression was detected in some cells in the immature alveolar walls and many cells were negative (Fig. $7 U$ and $V$ ). In the retinoidtreated group, intense signal for elastin mRNA localized to

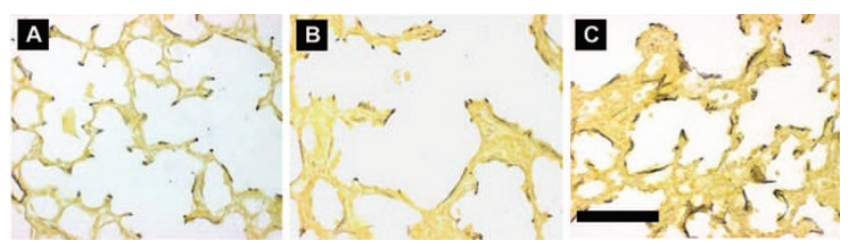

Figure 5. Elastic fibers in alveolar walls. Shown are peripheral lung specimens stained with Hart's elastic fiber stain (brown to black) and counterstained with tartrazine (yellow) at $200 \times$ magnification. (A) 140-d gestational control specimens exhibit dense and focal elastic fibers localizing primarily to the tips of alveolar walls. (B) Specimens from the ventilation-only group exhibit focal and dense elastic fibers at sites where alveolar walls normally extend and along and within thickened alveolar walls. $(C)$ In lung specimens from the retinoid-treated group, marked thickening of saccular walls was often noted and dense accumulations of elastic fibers were found at sites along saccular walls. Bar $=100 \mu \mathrm{M}$.

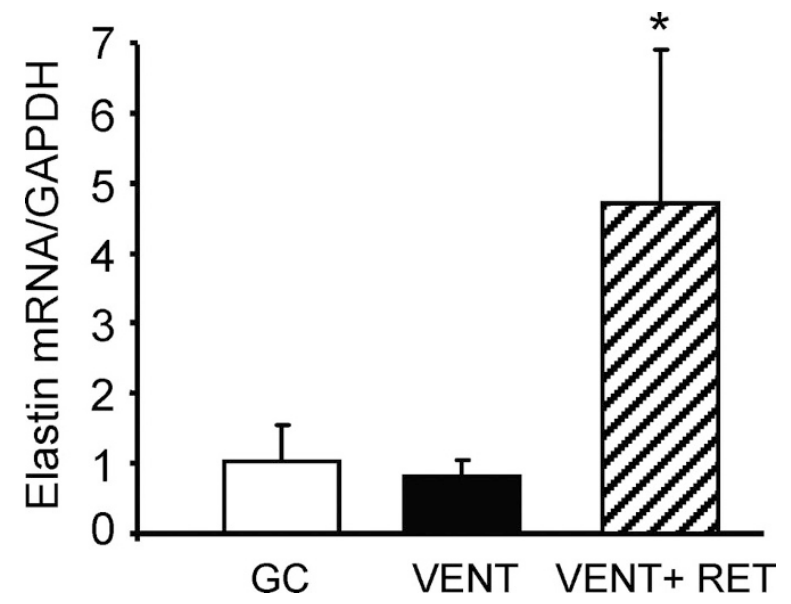

Figure 6. Retinoid treatment increases elastin expression in the ventilated preterm lung. Elastin expression in ventilated retinoid-treated lung specimens $(\mathrm{VENT}+\mathrm{RET})$ was increased compared with the ventilation-only group and gestational controls (GC).

individual cells in simplified alveolar walls (Fig. $7 W$ and $X$ ). These results are consistent with a marked induction of elastin expression with retinoid treatment, as indicated by the quantitative RT-PCR results.

Changes in $\alpha$-SMA staining with ventilation and retinoid treatment. The distribution of smooth muscle cells and alveolar myofibroblasts within the walls of terminal air spaces was assessed by staining for $\alpha$-SMA (Fig. 8). Cells intensely positive for $\alpha$-SMA were found at bends in alveolar walls and at the tips of alveolar walls in 140-d gestational control lungs (Fig. $8 A$ and $B$ ). In ventilated lungs with (Fig. $8 E$ and $F$ ) and without retinoid treatment (Fig. $8 C$ and $D$ ), intensely staining cells were found at obtuse angles of saccular walls with short, thick extensions. Cells with intermediate and weak but positive intensity staining for $\alpha$-SMA were abundant in both ventilated treatment groups. Quantitative analysis of these subpopulations showed that cells weakly positive for $\alpha$-SMA were more abundant in the ventilated treatment groups (Fig. $8 G)$. This population may represent cells transitioning to a smooth muscle-like phenotype in the ventilated premature lung. 

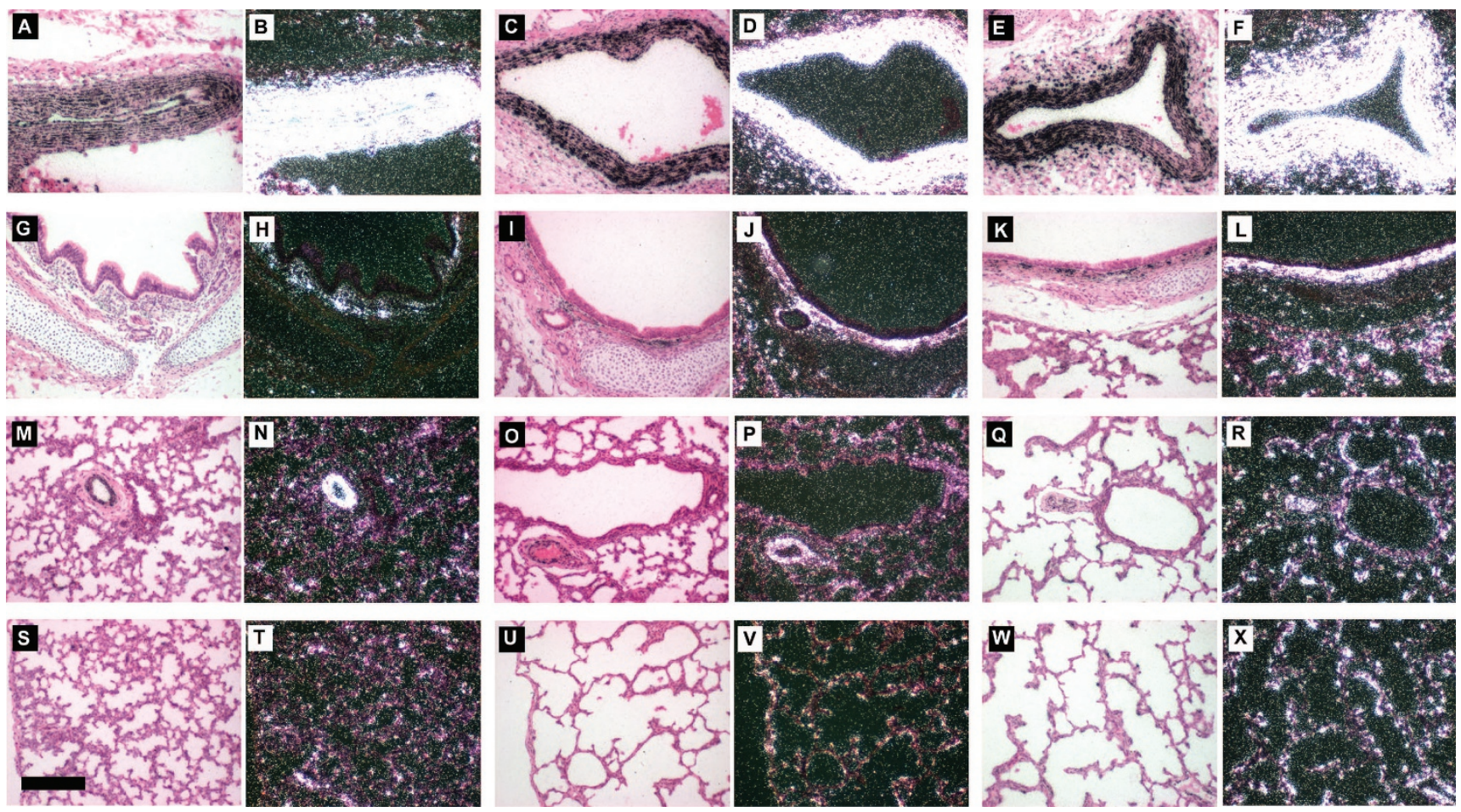

Figure 7. Localization of elastin expression in premature baboon lung. Shown are paired bright- and dark-field views of sections of lung hybridized in situ for tropoelastin mRNA. Signal appears as white dots in the dark-field views. $(A-F)$ Intralobar pulmonary arteries. $(G-L)$ Largest airways. $(M-R)$ Paired conducting airways and arteries. $(S-X)$ Peripheral lung sections. $(A, B, G, H, M, N, S, T)$ From the 140 -d gestational controls. $(C, D, I, J, O, P, U, V)$ From the ventilation-only group. ( $E, F, K, L, Q, R, W, X)$ From the retinoid supplementation group.

\section{DISCUSSION}

Angiogenesis and elastic fiber formation are key factors in alveolar development that may be altered in BPD (25), and both these pathways are retinoid sensitive $(18,26)$. Retinoid supplementation is the only treatment to date that increases alveolar number in experimental models (3) and has also been shown to reduce the incidence or severity of BPD in some clinical trials (6). The clinical benefit is a reduction in the combined outcome of oxygen requirement at 36-wk gestational age or reduced mortality for one of every 14 or 15 premature infants receiving vitamin A treatment compared with controls (9). We therefore studied the effects of retinoid supplementation on these parameters and on lung physiology in a primate model of BPD.

Physiologic parameters were not improved by vitamin A supplementation, and the incidence of early death was greater in the vitamin A-supplemented group (1/5) compared with the placebo-treated group. A longer treatment period, altered dose (25), or increasing the number of animals per treatment group may have resulted in a detected benefit of vitamin A supplementation in this model. The results of this study in a primate model are in line with findings that retinoid supplementation may benefit a small subset of infants at risk of BPD.

Retinoid supplementation has yielded variable results in other animal models of BPD $(5,27,28)$, with the most marked effects in rodent models $(3,29)$. It seems unlikely that the antenatal steroid treatment blocked a potential benefit of retinoid supplementation because antenatal steroid therapy was common in studies in which retinoid supplementation showed some benefit in premature infants (9). Furthermore, retinoid treatment advanced alveolar development in a rodent model in which alveolar maturation was inhibited by postnatal glucocorticoid supplementation (3). These differences suggest that mechanical ventilation of the premature lung promotes signals that are not normalized by retinoid supplementation.

Retinoic acid treatment failed to correct the abnormal microvasculature of terminal air space walls noted previously in this model of BPD (11) or to enhance VEGF expression compared with untreated controls. Reports on the effects of retinoids on angiogenesis vary widely. Retinoic acid increases VEGF expression in lung epithelial cells (14) and stimulates fetal pulmonary microvascular endothelial cell proliferation in vitro (23). Retinoids reverse dexamethasone-mediated repression of lung VEGF receptor 2 in newborn mice (30) and restore alveolarization caused by pharmacologic blockade of this receptor (23). In contrast, retinoids arrest angiogenesis in certain tumor subtypes (31) and reduce endothelial cell number and decrease vessel branching in an in vivo model of angiogenesis (32). The failure of retinol treatment to prevent abnormal capillaries in this study may be due to dose, timing, or the complex interplay of cell events that are necessary for vascular development.

In contrast to increased elastin expression and fiber deposition in a lamb model of BPD (33), lung elastin mRNA expression was modestly decreased in 125 -d gestation baboons delivered prematurely and ventilated $14 \mathrm{~d}$ compared with 140-d gestational control animals. This difference may represent what occurs in the "new BPD" found in premature neonates treated with antenatal steroids, surfactant upon birth, and low tidal volume ventilation. Retinoid supplementation of 

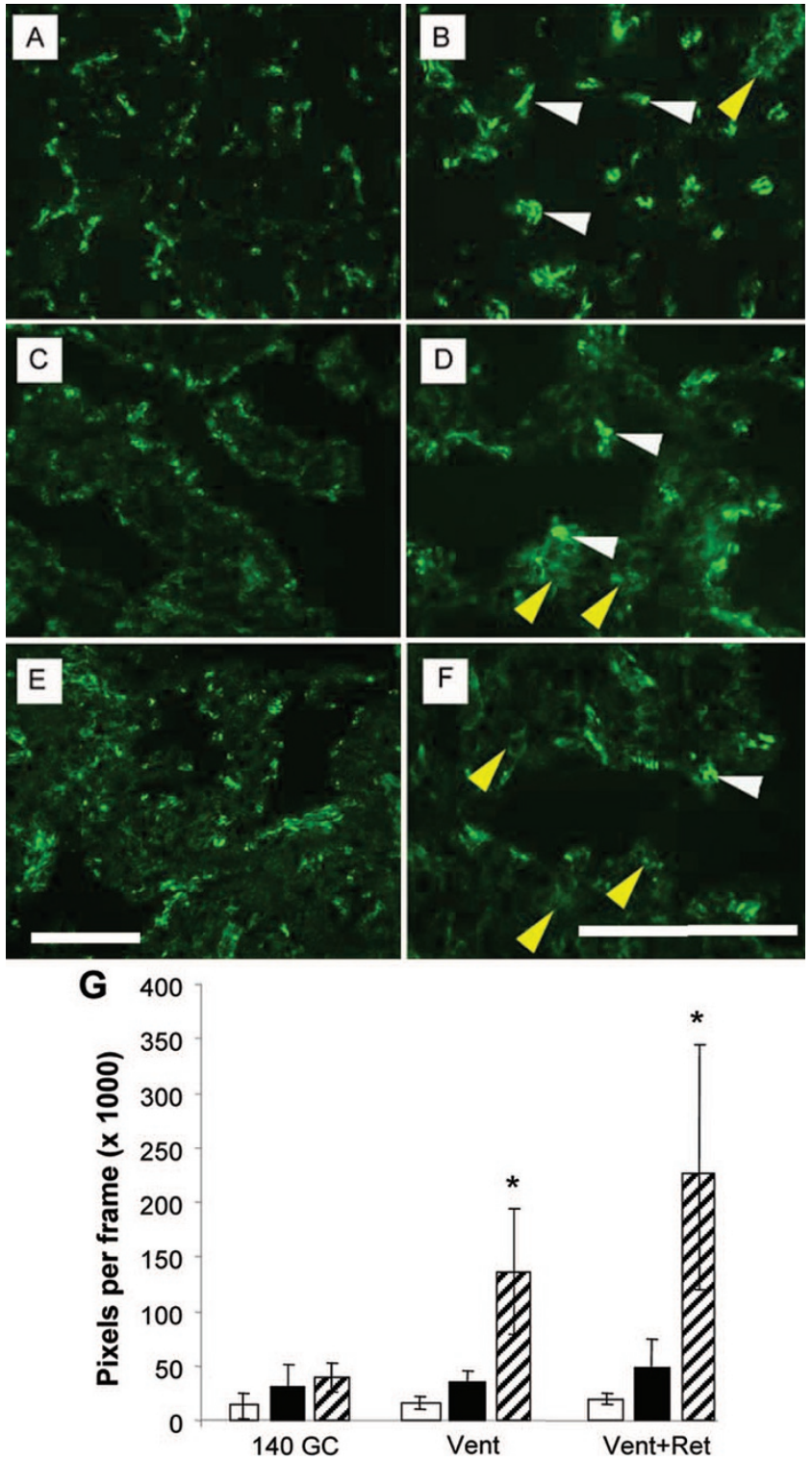

Figure 8. Staining for $\alpha$-SMA is altered by ventilation of premature lung. Shown are peripheral lung sections stained by immunofluorescence for $\alpha$-SMA at $\times 200(A, C, E)$ and $\times 400(B, D, F) .(A, B) 140$-d gestational control lung with intense staining (bright green signal, white arrowheads) of small cells in alveolar walls. Few positive cells exhibit intermediate staining (faint green signal) or are large (yellow arrowhead). $(C, D)$ In the ventilationonly group, intensely staining cells do not localize to tips of emerging alveolar septae. More cells exhibit less intense staining and larger size (yellow arrowheads). $(E, F)$ In the retinoid treatment group, cells of both phenotypes are also found. $(G)$ Quantification of area occupied by brightly staining cells (open columns), cells staining with medium intensity (solid columns), and cells slightly positive for $\alpha$-SMA (hatched columns). ${ }^{*} p<0.05$.

the ventilated preterm neonate increased lung elastin expression, particularly in cells that are positive for $\alpha$-SMA in saccular and alveolar walls. This localization is similar to findings in a neonatal rat model of retinoid supplementation during hyperoxia (5). Thibeault et al. (34) showed that elastic fiber density is increased in premature infants who died of chronic lung disease after prolonged mechanical ventilation (mean of $29 \mathrm{~d}$ ), but elastin expression was not quantified or localized in that study.
Mechanical ventilation of the premature lung resulted in a larger number of cells expressing $\alpha$-SMA staining in the walls of terminal air spaces. In ventilated lungs, cells of larger size staining intensely for $\alpha$-SMA were found in alveolar walls of ventilated lungs along with cells with intermediate staining intensity. This suggests that there is recruitment of other cell types to a myofibroblast-like phenotype in the ventilated premature lung. The abnormal distribution of elastic fibers in the BPD lung may result from expression and synthesis of elastin by cells with this intermediate phenotype.

An important finding of this study is that altering alveolar elastin expression through treatment with retinoids did not result in improved alveolar development. Positively affecting one system important in alveolar development, without improving angiogenesis, may fail to result in improved alveolar development.

\section{REFERENCES}

1. Northway WH Jr 1992 An introduction to bronchopulmonary dysplasia. Clin Perinatol 19:489-495.

2. Jobe AJ 1999 The new BPD: an arrest of lung development. Pediatr Res 46:641643

3. Massaro GD, Massaro D 1996 Postnatal treatment with retinoic acid increases the number of pulmonary alveoli in rats. Am J Physiol 270:L305-L310

4. Kaza AK, Kron IL, Kern JA, Long SM, Fiser SM, Nguyen RP, Tribble CG, Laubach VE 2001 Retinoic acid enhances lung growth after pneumonectomy. Ann Thorac Surg 71:1645-1650

5. Veness-Meehan KA, Pierce RA, Moats-Staats BM, Stiles AD 2002 Retinoic acid attenuates O2-induced inhibition of lung septation. Am J Physiol Lung Cell Mol Physiol 283:L971-L980

6. Ambalavanan N, Wu TJ, Tyson JE, Kennedy KA, Roane C, Carlo WA 2003 A comparison of three vitamin A dosing regimens in extremely-low-birth-weight infants. J Pediatr 142:656-661

7. D'Angio CT, Maniscalco WM 2004 Bronchopulmonary dysplasia in preterm infants: pathophysiology and management strategies. Paediatr Drugs 6:303-330

8. Mentro AM 2004 Vitamin A and bronchopulmonary dysplasia: research, issues, and clinical practice. Neonatal Netw 23:19-23

9. Tyson JE, Wright LL, Oh W, Kennedy KA, Mele L, Ehrenkranz RA, Stoll BJ, Lemons JA, Stevenson DK, Bauer CR, Korones SB, Fanaroff AA 1999 Vitamin A supplementation for extremely-low-birth-weight infants. National Institute of Child Health and Human Development Neonatal Research Network. N Engl J Med 340:1962-1968

10. Bhatt AJ, Pryhuber GS, Huyck H, Watkins RH, Metlay LA, Maniscalco WM 2001 Disrupted pulmonary vasculature and decreased vascular endothelial growth factor, Flt-1, and TIE-2 in human infants dying with bronchopulmonary dysplasia. Am J Respir Crit Care Med 164:1971-1980

11. Maniscalco WM, Watkins RH, Pryhuber GS, Bhatt A, Shea C, Huyck H 2002 Angiogenic factors and alveolar vasculature: development and alterations by injury in very premature baboons. Am J Physiol Lung Cell Mol Physiol 282:L811-L823

12. De Paepe ME, Mao Q, Powell J, Rubin SE, Dekoninck P, Appel N, Dixon M, Gundogan F 2006 Growth of pulmonary microvasculature in ventilated preterm infants. Am J Respir Crit Care Med 173:204-211

13. Abman SH 2001 Bronchopulmonary dysplasia: "a vascular hypothesis." Am J Respir Crit Care Med 164:1755-1756

14. Maeno T, Tanaka T, Sando Y, Suga T, Maeno Y, Nakagawa J, Hosono T, Sato M, Akiyama H, Kishi S, Nagai R, Kurabayashi M 2002 Stimulation of vascular endothelial growth factor gene transcription by all trans retinoic acid through $\mathrm{Sp} 1$ and $\mathrm{Sp} 3$ sites in human bronchioloalveolar carcinoma cells. Am J Respir Cell Mol Biol 26:246-253

15. Lai L, Bohnsack BL, Niederreither K, Hirschi KK 2003 Retinoic acid regulates endothelial cell proliferation during vasculogenesis. Development 130:64656474

16. Cho DH, Choi YJ, Jo SA, Nam JH, Jung SC, Jo I 2005 Retinoic acid decreases nitric oxide production in endothelial cells: a role of phosphorylation of endothelial nitric oxide synthase at Ser (1179). Biochem Biophys Res Commun 326:703-710

17. McGowan S, Jackson SK, Jenkins-Moore M, Dai HH, Chambon P, Snyder JM 2000 Mice bearing deletions of retinoic acid receptors demonstrate reduced lung elastin and alveolar numbers. Am J Respir Cell Mol Biol 23:162-167

18. McGowan SE, Doro MM, Jackson SK 1997 Endogenous retinoids increase perinatal elastin gene expression in rat lung fibroblasts and fetal explants. Am J Physiol 273:L410-L416

19. Shifren A, Mecham RP 2006 The stumbling block in lung repair of emphysema: elastic fiber assembly. Proc Am Thorac Soc 3:428-433

20. McCurnin DC, Pierce RA, Chang LY, Gibson LL, Osborne-Lawrence S, Yoder BA, Kerecman JD, Albertine KH, Winter VT, Coalson JJ, Crapo JD, Grubb PH, Shau PW 2005 Inhaled NO improves early pulmonary function and modifies lung growth 
and elastin deposition in a baboon model of neonatal chronic lung disease. Am J Physiol Lung Cell Mol Physiol 288:L450-L459

21. Shifren A, Durmowicz AG, Knutsen RH, Hirano E, Mecham RP 2006 Elastin protein levels are a vital modifier affecting normal lung development and susceptibility to emphysema. Am J Physiol Lung Cell Mol Physiol 292(3):L778-L787

22. Coalson JJ, Winter VT, Siler-Khodr T, Yoder BA 1999 Neonatal chronic lung disease in extremely immature baboons. Am J Respir Crit Care Med 160:1333-1346

23. Cho SJ, George CL, Snyder JM, Acarregui MJ 2005 Retinoic acid and erythropoietin maintain alveolar development in mice treated with an angiogenesis inhibitor. Am J Respir Cell Mol Biol 33:622-628

24. Lachgar S, Charveron M, Gall Y, Bonafe JL 1999 Inhibitory effects of retinoids on vascular endothelial growth factor production by cultured human skin keratinocytes. Dermatology 199:25-27

25. Bourbon J, Boucherat O, Chailley-Heu B, Delacourt C Control mechanisms of lung alveolar development and their disorders in bronchopulmonary dysplasia. Pediatr Res. 57:38R-46R, 2005.

26. McGowan SE Contributions of retinoids to the generation and repair of the pulmonary alveolus. Chest. 121:206S-208S, 2002.

27. Willet KE, Jobe AH, Ikegami M, Newnham J, Sly PD 2000 Antenatal retinoic acid does not alter alveolization or postnatal lung function in preterm sheep. Eur Respir J $16: 101-107$
28. Veness-Meehan KA, Bottone FG Jr, Stiles AD 2000 Effects of retinoic acid on airspace development and lung collagen in hyperoxia-exposed newborn rats. Pediatr Res 48:434-444

29. Massaro GD, Massaro D 2000 Retinoic acid treatment partially rescues failed septation in rats and in mice. Am J Physiol Lung Cell Mol Physiol 278:L955-L960

30. Clerch LB, Baras AS, Massaro GD, Hoffman EP, Massaro D 2004 DNA microarray analysis of neonatal mouse lung connects regulation of KDR with dexamethasoneinduced inhibition of alveolar formation. Am J Physiol Lung Cell Mol Physiol 286:L411-L419

31. Lingen MW, Polverini PJ, Bouck NP 1998 Retinoic acid and interferon alpha act synergistically as antiangiogenic and antitumor agents against human head and neck squamous cell carcinoma. Cancer Res 58:5551-5558

32. Suzuki Y, Komi Y, Ashino H, Yamashita J, Inoue J, Yoshiki A, Eichmann A, Amanuma H, Kojima S 2004 Retinoic acid controls blood vessel formation by modulating endothelial and mural cell interaction via suppression of Tie 2 signaling in vascular progenitor cells. Blood 104:166-169

33. Pierce RA, Albertine KH, Starcher BC, Bohnsack JF, Carlton DP, Bland RD 1997 Chronic lung injury in preterm lambs: disordered pulmonary elastin deposition. Am J Physiol 272:L452-L460

34. Thibeault DW, Mabry SM, Ekekezie II, Truog WE 2000 Lung elastic tissue maturation and perturbations during the evolution of chronic lung disease. Pediatrics 106:1452-1459 